\title{
Isolation, Characterization and Identification of Probiotic Lactic Acid Bacteria (LAB) from Honey Bees
}

\author{
M. Mathialagan ${ }^{1 *}$, Y.S. Johnson Thangaraj Edward ${ }^{1}$, P.M.M. David ${ }^{2}$, \\ M. Senthilkumar ${ }^{3}$, M.R. Srinivasan ${ }^{1}$ and S. Mohankumar ${ }^{1}$ \\ ${ }^{1}$ Department of Agricultural Entomology, ${ }^{3}$ Department of Agricultural Microbiology, Tamil \\ Nadu Agricultural University, Coimbatore, Tamil Nadu, India \\ ${ }^{2}$ Department of Agricultural Entomology, Agricultural College and Research Institute, \\ Killikulam, Tamil Nadu, India \\ *Corresponding author
}

\section{A B S T R A C T}

\begin{tabular}{|l|}
\hline Ke y w or d s \\
$\begin{array}{l}\text { Probiotic lactic acid } \\
\text { bacteria (LAB), } \\
\text { Diversity, Honey bee } \\
\text { species }\end{array}$ \\
\hline Article Info \\
\hline $\begin{array}{l}\text { Accepted: } \\
\text { 07 March } 2018 \\
\text { Available Online: } \\
\text { 10 April } 2018\end{array}$ \\
\hline
\end{tabular}

\section{Introduction}

Lactic acid bacteria (LAB) are a group of Gram-positive, non-spore forming coccior rods which produce lactic acid as the major end product of the fermentation of carbohydrates (Axelsson, 1998). They are generally recognized as safe (GRAS) food grade microorganisms exploited as probiotics that confer health benefits on the host (FAO/WHO, 2001). Commensals within humans, insects and animals, these bacteria are common inhabitants of guts. Species of the
Laboratory experiments were conducted to explore the diversity of naturally-occurring probiotic lactic acid bacteria (LAB) associated with honey bees and to understand whether they can be used as probiotics to manage honey bee stressors. Honey stomach, honey, bee bread, bee pollen and royal jelly from different species of honey bees (Apis ceranaindica Fabricius, Apis melliefra Linnaeus, Apis florea Fabricius, Apis dorsata Fabricius, Tetragonula iridipennis Smith) were examined for the presence of LAB. The results indicated a rich diversity of LAB in the samples analysed, with 42 isolates belonging to six genera, viz., Enterococcus (23.8\%), Micrococcus (18.8\%), Streptococcus (13.8 \%), Pediococcus (13.8 \%), Lactobacillus (10.0\%), Lactococcus (10.0\%) and Leuconostoc (10.0\%). Their scope in disease management is discussed. 
Bifidobacterium within the crop in biofilms (Vasquez et al., 2012), with potential to inhibit American Foul Brood-causing Paenibacillus larvae growth on agar plate (Forsgren et al., 2009). Especially, Lactobacullus kunkeei is predominantly found in A. laboriosa honey in Nepal $\left(10^{8} \mathrm{cfu}\right.$ per gram honey), followed by A. mellifera honey in Africa. This fructophilic, osmotolerant and acid-resistant bacterium originates from the flower nectar and/or pollen grains collected and ingested by the bees and are found in honey bees in stored pollen and honey (Endo et al., 2012; Rangberg et al., 2012; Corby-Harris et al., 2014). This species was also predominant in the honey stomach of Apisdorsata (Tajabadi et al., 2011). Now-adays honey bees all over the world are reported to be in stress, leading to colony collapse disorder (CCD) (Ellis et al., 2015). Among the stressors, viral diseases, especially Thai sacbrood (TSBV), European foulbrood (EFB) and the parasitic mite Varroajacobsoni (Oudemans) -associated diseases (varroosis), are important in Indian bees, Apis cerana (Rao et al., 2016; Mathialagan et al., 2017). In the absence of effective control measures and in view of antibiotics residues in honey, alternative strategies are needed to control them. Prophylactic feeding of probiotic LAB isolated from the honey bee environment may help the bees overcome the stress and sustain their health. Therefore this study was undertaken to isolate, characterize and identify the diversity of LAB species from honey stomach, honey, bee pollen, bee bread and royal jelly.

\section{Materials and Methods}

\section{Collection of samples}

Laboratory investigations were conducted at the Department of Agricultural Microbiology, Tamil Nadu Agricultural University, Coimbatore during 2016-2017. Workers of the Indian bee (Apis ceranaindica Fab.), Italian bee (Apis mellifera Lin.), Little bee (Apis florea Fab.), Rock bee (Apis dorsata Fab.) and Dammer bee (Tetragonula iridipennis Smith) were collected alive from different places. They were kept in a refrigerator freezer for five minutes before they were dissected to detach the honey stomach (crop) from the abdomen (Plate 1). The detached crop was transferred to a sterilized Eppendorf tube containing $1.0 \mathrm{ml}$ sterile water and crushed for LAB isolation. Unprocessed honey samples were collected from the feral colonies of $A$. ceranaindica, A. mellifera A. florea, A. dorsataand $T$. iridipennis (Plate 2). Workers of $A$. ceranaindica were collected to extract the royal jelly by carefully pressing the head of the nurse bees (Plate 3). Fresh royal jelly was scooped out from the queen cell cups of the Indian bees (Plate 4). Commercially available dry royal jelly sample was donated by a firm (Plate 5). Samples were collected using a micropipette and dissolved in $1.5 \mathrm{ml}$ sterile water in a sterilized Eppendorf tube. Bee bread samples were collected from various bee species (A. ceranaindica, $A$. mellifera, A. florea, A. dorsata and $T$. iridipennis) with the help of a sterilized micropipette tip (Plate 6). Commercial bee pollen of $A$. mellifera was received as a donation (Plate 7). Foraging workers of different bee species were collected from the hives to collect pollen grains from the hind legs. They were transferred to a $1.0 \mathrm{ml}$ Eppendorf tube before serial dilution and plating in the laboratory.

\section{Isolation and enumeration of LAB}

LAB populations were enumerated by serial dilution and plate count technique with $1.0 \mathrm{ml}$ sample serially diluted to $10^{-5}$ dilutions before plating in Lactobacillus MRS Agar medium for isolation (Awan and Rahman, 2005). Calcium carbonate $\left(\mathrm{CaCO}_{3}\right)$ was added to MRS medium to induce more LAB growth (Aween et al., 2012). Cycloheximide was 
added to the above medium to avoid fungal contamination. The plates were incubated at $30 \pm 2{ }^{\circ} \mathrm{C}$. The colonies that matured in 3 days were counted and expressed as Colony Forming Units (CFU / ml). Morphologically distinct and well isolated colonies were picked and transferred to new MRS agar plates by streaking to obtain pure colonies that were maintained on glycerol stocks. The cultures were stored at $-80^{\circ} \mathrm{C}$ for further studies. Gram staining was done as per the protocol described by Sharma (2007). The stained slides were examined under themicroscope.

\section{Identification of the isolates}

All the isolates were tested for Gram reaction and catalase test as described by Harrigan and McCance (1976). The isolates were then identified with reference to Bergey's Manual of Determinative Bacteriology. Identification was based mainly on gram staining, absence of catalase, cultural and morphological characteristics (such as its elevation, shape, colour and texture of the colonies). Lactobacilli species were taxonomically classified following the discriminatory schemes of Kandler and Weiss (1986), Hammes et al., (1992) and Liu et al., (2014).

\section{Results and Discussion}

A random survey of honey bee niches for the presence of LAB indicated that different species of LAB are commonly associated with A. ceranaindica, A. mellifera, A. florea, A. dorsata and $T$. iridipennis. As many as 42 LAB isolates were isolated from the honey stomach, honey, bee pollen, bee bread and royal jelly collected from different sources and their morphological details with population densities are presented in Tables 1, 2, 3, 4 and 5. Morphologically, most isolates $(\mathrm{N}=34)$ were cocci and the rest $(\mathrm{N}=8)$ rod-shaped, grouped into seven LAB genera, namely, Enterococcus (23.8 \%), Micrococcus (18.8\%),
Streptococcus (13.8\%), Pediococcus (13.8\%), Lactobacillus (10.0\%), Lactococcus (10.0\%) and Leuconostoc (10.0\%) (Figure 1). Their populations varied from $16.40-35.87 \times 10^{5}$ $\mathrm{CFU} / \mathrm{ml}$ in honey stomach (Table 1), 10.43 $31.30 \times 10^{5} \mathrm{CFU} / \mathrm{ml}$ in honey (Table 2), 5.14 $14.35 \times 10^{5} \mathrm{CFU} / \mathrm{ml}$ in bee pollen (Table 3 ), $13.05-25.15 \times 10^{5} \mathrm{CFU} / \mathrm{ml}$ in bee bread (Table 4) and $10.23-20.15 \times 10^{5} \mathrm{CFU} / \mathrm{ml}$ in royal jelly (Table 5). Heterofermentative, these LAB grew typically in MRS agar medium, producing protective exopolysaccharides. They were all gram positive, catalase negative and non-spore forming rods and cocci in singles, pairs, chains or tetrads (Plate 8, 9, 10). Species from the LAB genera Pediococcus, Aerococcus, Tetrageono coccus are all capable of division in two planes and form tetrads (Holzapfel and Wood, 2014). Enterococcus in singles, pairs, small chains or small groups are cosmopolitan associated with insect gut, fermented foods and drinking water (Lebreton et al., 2014). Rod-shaped LAB were rarely found in the honey stomach and honey of $A$. ceranaindica, A. mellifera and A. dorsata and the bee pollen of A. ceranaindica at Coimbatore. Generally, LAB species may differ from place to place. Hosny et al., (2009) isolated several species of Lactobacillus, Streptococcus, Micrococcus, Enterococcus and Micrococcus from honey. Strains of Lactobacillus acidophilus were isolated from honey samples by Aween et al., (2012). Lactobacillus is commonly associated with honey bees even in India (Pattabhiramaiah et al., 2012). Elsewhere L. kunkeei has been reported as the most common fructophilic species (Vasquez et al., 2012). Earlier different species of bacteria have been reported from the pollen (CorbyHarris et al., 2014) and honey stomach (Tajabadi et al., 2011). Royal jelly from queen cell cups, nurse bee heads and a bee product of commerce also harboured LAB in them (10.23 $-20.15 \times 10^{5} \mathrm{CFU} / \mathrm{ml}$ ). Isolation of LAB from the fourth instar larval gut in this study 
assumes significance as the larval gut is the target of highly pathogenic bacteria and fungi. Both royal jelly and worker jelly (bee bread + gland secretions + diluted honey) play an important role in the nature of bacterial composition as reported by Vojvodic et al., (2013) who found that first and second larval instars contained almost exclusively Acetobacteraceae, while later instars were dominated by one or two very different Lactobacillus spp. Acetobacteraceae includes acetic acid bacteria (AAB), a large group of obligate aerobic gram-negative bacteria, well adapted to sugary and alcoholised fluid such as vinegar, fruit juice, sap water, alcoholic beverages and flowers. AAB of the genera Gluconobacter, Acetobacter, Gluconacetobacter and Saccharibacter have been reported as symbionts of bees that may also regulate the innate immune system homeostasis in insects (Crotti et al., 2010). The sugar-loving and flower-associated Gluconobacters are among the predominant bacterial groups in bees (Hamdi et al., 2011). While both $\mathrm{AAB}$ and $\mathrm{LAB}$ may play a role in nutrient processing in adult bees, they contribute to larval immunity during the early and fragile stage of honey bee development (Vojvodic et al., 2013). Royal jelly is considered highly antimicrobial, possessing a $\mathrm{pH}$ between 3.6 and 4.2 , with many peptides active against gram-positive and gramnegative bacteria, fungi and yeasts. However, LAB isolated from royal jelly in this investigation may be related to its water content which is around 67 per cent (Wongchai and Ratanavalachai, 2002).

Frequent occurrence of LAB associated with honey bees strengthens the need to exploit them in honey bee health management strategy. Symbionts play a major role in maintaining the health of the host animal, including insects, especially honey bees. LAB is a key symbiotic micro-organism in bees, providing not only adequate nutrition but also host immunity against pathogens (Vasquez et al., 2012). At present, acaricides and antibiotics are extensively used in apiculture to manage the ectoparasitic Varroa mite syndrome. This leads to resistance in mites and pathogens (Thomson et al., 2002; Berry et al., 2013).

Fig.1 Population density and percentage of isolates identified in the survey from different genera of lactic acid bacteria (LAB). Vertical bars indicate the standard error. CFU, colony forming units

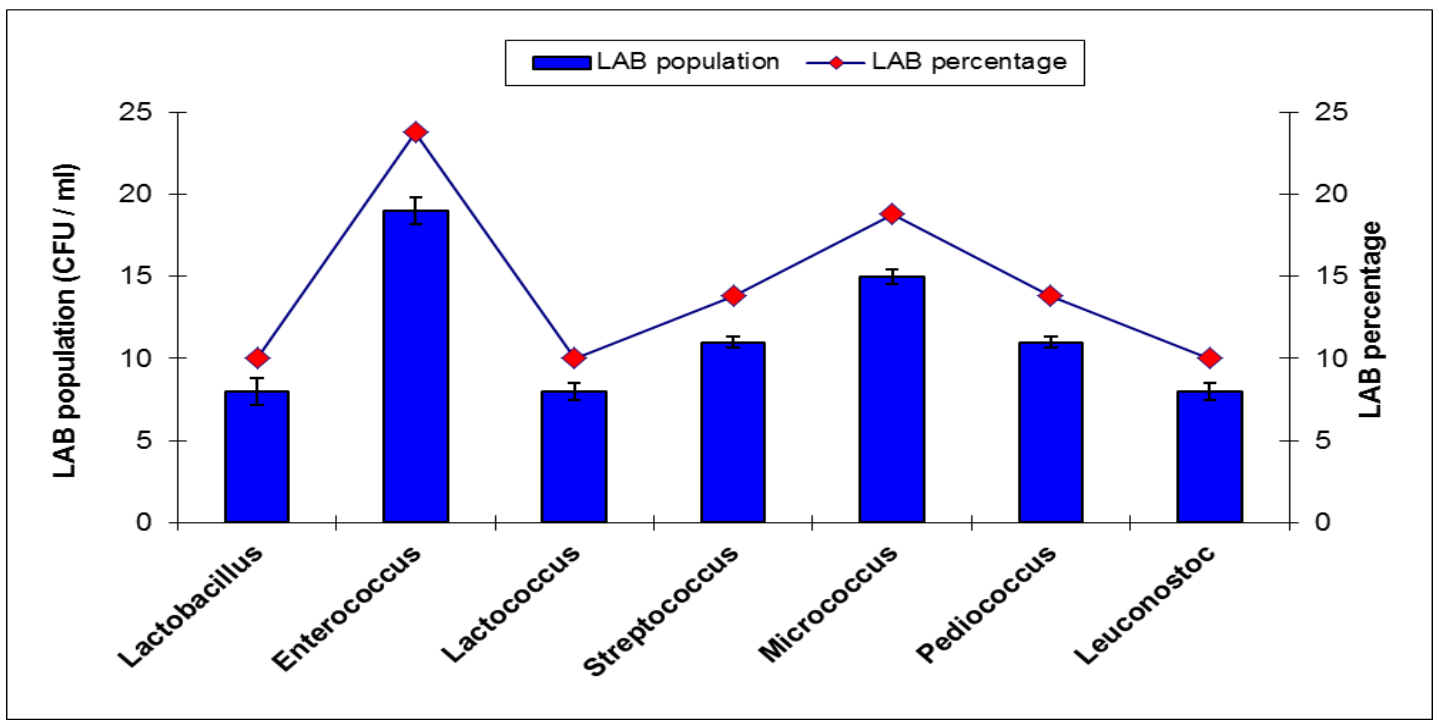


Table.1 Population and colony morphology of LAB in the honey stomach of honey bees

\begin{tabular}{|c|c|c|c|c|c|c|c|c|c|c|}
\hline \multirow[t]{2}{*}{ Bee species } & \multirow{2}{*}{$\begin{array}{l}\text { Place of } \\
\text { collection }\end{array}$} & \multirow{2}{*}{$\begin{array}{l}\text { Isolation } \\
\text { source } \\
\text { (caste) }\end{array}$} & \multirow{2}{*}{$\begin{array}{l}\text { Number of } \\
\text { isolates }\end{array}$} & \multirow{2}{*}{$\begin{array}{c}\text { LAB } \\
\left(10^{5} \mathrm{CFU} / \mathrm{ml}\right)\end{array}$} & \multicolumn{4}{|c|}{ Colony morphology } & \multirow[t]{2}{*}{ Cell shape } & \multirow{2}{*}{$\begin{array}{c}\text { Probable } \\
\text { LAB genera }\end{array}$} \\
\hline & & & & & Elevation & Colony texture & Edge & $\begin{array}{l}\text { Colony } \\
\text { colour }\end{array}$ & & \\
\hline \multirow[t]{5}{*}{ A. ceranaindica } & \multirow[t]{5}{*}{ TNAU } & \multirow[t]{5}{*}{$\begin{array}{l}\text { Worker } \\
\text { (healthy) }\end{array}$} & $\begin{array}{l}\text { Honey } \\
\text { stomach1 }\end{array}$ & $\begin{array}{c}35.87 \pm 10.76 \\
(n=3)\end{array}$ & Raised & Coarse surface & Smooth & Whitish & Rods & Lactobacillus \\
\hline & & & $\begin{array}{c}\text { Honey } \\
\text { stomach2 }\end{array}$ & $\begin{array}{c}29.71 \pm 8.54 \\
\quad(n=3)\end{array}$ & Flat & Shinny surface & Rough & Yellowish & Rods & Lactobacillus \\
\hline & & & $\begin{array}{c}\text { Honey } \\
\text { stomach3 }\end{array}$ & $\begin{array}{c}17.21 \pm 5.90 \\
(n=3)\end{array}$ & Flat & Coarse surface & Rough & Creamy & Cocci in singles & $\begin{array}{c}\text { Enterococcus } \\
\text { Lactococcus } \\
\text { Leuconostoc }\end{array}$ \\
\hline & & & $\begin{array}{c}\text { Honey } \\
\text { stomach4 }\end{array}$ & $\begin{array}{l}15.41 \pm 4.76 \\
(n=3)\end{array}$ & Raised & $\begin{array}{l}\text { Shinny mucoid } \\
\text { surface }\end{array}$ & Smooth & Creamy & Cocci in chains & $\begin{array}{c}\text { Enterococcus } \\
\text { Streptococcus } \\
\text { Pediococcus }\end{array}$ \\
\hline & & & $\begin{array}{c}\text { Honey } \\
\text { stomach5 }\end{array}$ & $\begin{array}{c}10.91 \pm 2.90 \\
(n=3)\end{array}$ & Flat & Shinny surface & Smooth & Whitish & Cocci in tetrads & Micrococcus \\
\hline \multirow[t]{2}{*}{ A. ceranaindica } & \multirow[t]{2}{*}{ TNAU } & \multirow[t]{2}{*}{$\begin{array}{l}\text { Worker } \\
\text { (Diseased) }\end{array}$} & $\begin{array}{l}\text { Honey } \\
\text { stomach1 }\end{array}$ & $\begin{array}{c}13.15 \pm 2.25 \\
\quad(n=3)\end{array}$ & Flat & Coarse surface & Rough & Creamy & Cocci in singles & $\begin{array}{c}\text { Enterococcus } \\
\text { Lactococcus } \\
\text { Leuconostoc }\end{array}$ \\
\hline & & & $\begin{array}{c}\text { Honey } \\
\text { stomach2 }\end{array}$ & $\begin{array}{c}9.50 \pm 4.75 \\
\quad(n=3)\end{array}$ & Raised & $\begin{array}{l}\text { Shinny mucoid } \\
\text { surface }\end{array}$ & Smooth & Creamy & Cocci in chains & $\begin{array}{c}\text { Enterococcus } \\
\text { Streptococcus } \\
\text { Pediococcus }\end{array}$ \\
\hline A. florea & TNAU & $\begin{array}{l}\text { Worker } \\
\text { (healthy) }\end{array}$ & $\begin{array}{l}\text { Honey } \\
\text { stomach1 }\end{array}$ & $\begin{array}{c}16.40 \pm 5.65 \\
(n=3)\end{array}$ & Flat & Shinny surface & Smooth & Whitish & Cocci in tetrads & Micrococcus \\
\hline \multirow[t]{2}{*}{ A. mellifera } & \multirow[t]{2}{*}{ TNAU } & \multirow[t]{2}{*}{$\begin{array}{l}\text { Worker } \\
\text { (healthy) }\end{array}$} & $\begin{array}{l}\text { Honey } \\
\text { stomach1 }\end{array}$ & $\begin{array}{c}24.67 \pm 7.54 \\
\quad(n=3)\end{array}$ & Flat & Shinny surface & Smooth & Whitish & Cocci in tetrads & Micrococcus \\
\hline & & & $\begin{array}{c}\text { Honey } \\
\text { stomach2 }\end{array}$ & $\begin{array}{c}16.73 \pm 5.64 \\
\quad(n=3)\end{array}$ & Raised & Coarse surface & Smooth & Whitish & Rods & Lactobacillus \\
\hline A.dorsata & TNAU & $\begin{array}{l}\text { Worker } \\
\text { (healthy) }\end{array}$ & $\begin{array}{l}\text { Honey } \\
\text { stomach1 }\end{array}$ & $\begin{array}{c}21.31 \pm 3.80 \\
\quad(n=3)\end{array}$ & Flat & Shinny surface & Rough & Yellowish & Rods & Lactobacillus \\
\hline T. iridipennis & TNAU & $\begin{array}{l}\text { Worker } \\
\text { (healthy) }\end{array}$ & $\begin{array}{l}\text { Honey } \\
\text { stomach1 }\end{array}$ & $\begin{array}{c}14.78 \pm 2.76 \\
(n=3)\end{array}$ & Flat & Shinny surface & Smooth & Whitish & Cocci in tetrads & Micrococcus \\
\hline
\end{tabular}

(Mean $\pm \mathrm{SE} ; \mathrm{SE}$, standard error; $\mathrm{n}=$ number of replicates; $\mathrm{CFU}$, colony forming units) 
Table.2 Population and colony morphology of LAB in the unprocessed comb honey of honey bee species

\begin{tabular}{|c|c|c|c|c|c|c|c|c|c|}
\hline \multirow[t]{2}{*}{ Bee species } & \multirow{2}{*}{$\begin{array}{l}\text { Place of } \\
\text { collection }\end{array}$} & \multirow{2}{*}{$\begin{array}{c}\text { Number of } \\
\text { isolates }\end{array}$} & \multirow{2}{*}{$\begin{array}{c}\text { LAB } \\
\left(10^{5} \mathrm{CFU} / \mathrm{ml}\right)\end{array}$} & \multicolumn{4}{|c|}{ Colony morphology } & \multirow[t]{2}{*}{ Cell shape } & \multirow{2}{*}{$\begin{array}{c}\text { Probable LAB } \\
\text { genera }\end{array}$} \\
\hline & & & & Elevation & Colony texture & Edge & $\begin{array}{l}\text { Colony } \\
\text { colour }\end{array}$ & & \\
\hline \multirow[t]{3}{*}{ A. ceranaindica } & \multirow[t]{3}{*}{ TNAU } & Honey 1 & $\begin{array}{c}31.30 \pm 6.61 \\
(n=3)\end{array}$ & Flat & Coarse surface & Rough & Creamy & Cocci in singles & $\begin{array}{l}\text { Enterococcus } \\
\text { Lactococcus } \\
\text { Leuconostoc }\end{array}$ \\
\hline & & Honey 2 & $\begin{array}{c}25.28 \pm 4.15 \\
(n=3)\end{array}$ & Raised & $\begin{array}{l}\text { Shinny mucoid } \\
\text { surface }\end{array}$ & Smooth & Creamy & Cocci in chains & $\begin{array}{c}\text { Enterococcus } \\
\text { Streptococcus } \\
\text { Pediococcus }\end{array}$ \\
\hline & & Honey 3 & $\begin{array}{c}17.37 \pm 3.90 \\
(\mathrm{n}=3)\end{array}$ & Raised & Coarse surface & Smooth & Whitish & Rods & Lactobacillus \\
\hline \multirow[t]{2}{*}{ A. mellifera } & \multirow[t]{2}{*}{ TNAU } & Honey 1 & $\begin{array}{c}18.20 \pm 5.51 \\
(n=3)\end{array}$ & Flat & Shinny surface & Smooth & whitish & Cocci in tetrads & Micrococcus \\
\hline & & Honey 2 & $\begin{array}{c}15.10 \pm 2.10 \\
(n=3)\end{array}$ & Flat & Shinny surface & Rough & Yellowish & Rods & Lactobacillus \\
\hline \multirow[t]{2}{*}{ A. florea } & \multirow[t]{2}{*}{ TNAU } & Honey 1 & $\begin{array}{c}12.09 \pm 2.41 \\
(n=3)\end{array}$ & Flat & Coarse surface & Rough & Creamy & Cocci in singles & $\begin{array}{l}\text { Enterococcus } \\
\text { Lactococcus } \\
\text { Leuconostoc }\end{array}$ \\
\hline & & Honey 2 & $\begin{array}{c}8.23 \pm 1.78 \\
(n=3)\end{array}$ & Raised & $\begin{array}{l}\text { Shinny mucoid } \\
\text { surface }\end{array}$ & Smooth & Creamy & Cocci in chains & $\begin{array}{c}\text { Enterococcus } \\
\text { Streptococcus } \\
\text { Pediococcus }\end{array}$ \\
\hline A.dorsata & TNAU & Honey 1 & $\begin{array}{c}15.37 \pm 5.80 \\
(n=3)\end{array}$ & Raised & Coarse surface & Smooth & Whitish & Rods & Lactobacillus \\
\hline T. iridipennis & TNAU & Honey 1 & $\begin{array}{c}10.43 \pm 4.62 \\
(n=3)\end{array}$ & Flat & Shinny surface & Smooth & whitish & Cocci in tetrads & Micrococcus \\
\hline
\end{tabular}

(Mean \pm SE of 3 replicates; SE, standard error; CFU, colony forming units) 
Table.3 Population and colony morphology of LAB in the bee pollen of honey bees

\begin{tabular}{|c|c|c|c|c|c|c|c|c|c|}
\hline \multirow[t]{2}{*}{ Bee species } & \multirow[t]{2}{*}{ Place of collection } & \multirow{2}{*}{$\begin{array}{c}\text { Number of } \\
\text { isolates }\end{array}$} & \multirow{2}{*}{$\begin{array}{c}\text { LAB } \\
\left(10^{5} \mathrm{CFU} / \mathrm{ml}\right)\end{array}$} & \multicolumn{4}{|c|}{ Colony morphology } & \multirow{2}{*}{$\begin{array}{c}\text { Cell } \\
\text { shape }\end{array}$} & \multirow{2}{*}{$\begin{array}{c}\text { Probable LAB } \\
\text { genera }\end{array}$} \\
\hline & & & & Elevation & $\begin{array}{l}\text { Colony } \\
\text { texture }\end{array}$ & Edge & $\begin{array}{l}\text { Colony } \\
\text { colour }\end{array}$ & & \\
\hline \multirow[t]{3}{*}{ A. ceranaindica } & \multirow{3}{*}{ TNAU } & Pollen 1 & $\begin{array}{c}14.35 \pm 2.31 \\
(n=3)\end{array}$ & Raised & $\begin{array}{l}\text { Shinny } \\
\text { mucoid } \\
\text { surface }\end{array}$ & Smooth & Creamy & Cocci in chains & $\begin{array}{c}\text { Enterococcus } \\
\text { Streptococcus } \\
\text { Pediococcus }\end{array}$ \\
\hline & & Pollen 2 & $\begin{array}{c}9.55 \pm 4.45 \\
(n=3)\end{array}$ & Flat & $\begin{array}{l}\text { Shinny } \\
\text { surface }\end{array}$ & Smooth & whitish & Cocci in tetrads & Micrococcus \\
\hline & & Pollen 3 & $\begin{array}{c}6.14 \pm 6.51 \\
(n=3)\end{array}$ & Raised & $\begin{array}{l}\text { Coarse } \\
\text { surface }\end{array}$ & Smooth & Whitish & Rods & Lactobacillus \\
\hline \multirow[t]{2}{*}{ A. mellifera } & TNAU & Pollen 1 & $\begin{array}{c}11.21 \pm 5.31 \\
(n=3)\end{array}$ & Flat & $\begin{array}{l}\text { Coarse } \\
\text { surface }\end{array}$ & Rough & Creamy & Cocci in singles & $\begin{array}{c}\text { Enterococcus } \\
\text { Lactococcus } \\
\text { Leuconostoc }\end{array}$ \\
\hline & $\begin{array}{l}\text { Commercial bee } \\
\text { pollen } \\
\text { (Dhalavaipuram) }\end{array}$ & Pollen 1 & $\begin{array}{c}10.30 \pm 2.86 \\
(n=3)\end{array}$ & Flat & $\begin{array}{l}\text { Shinny } \\
\text { surface }\end{array}$ & Smooth & whitish & Cocci in tetrads & Micrococcus \\
\hline \multirow[t]{2}{*}{ A. florea } & \multirow{2}{*}{ TNAU } & Pollen 1 & $\begin{array}{c}12.67 \pm 6.43 \\
(n=3)\end{array}$ & Flat & $\begin{array}{l}\text { Shinny } \\
\text { surface }\end{array}$ & Smooth & whitish & Cocci in tetrads & Micrococcus \\
\hline & & Pollen 2 & $\begin{array}{c}8.70 \pm 2.30 \\
(n=3)\end{array}$ & Raised & $\begin{array}{l}\text { Shinny } \\
\text { mucoid } \\
\text { surface }\end{array}$ & Smooth & Creamy & Cocci in chains & $\begin{array}{c}\text { Enterococcus } \\
\text { Streptococcus } \\
\text { Pediococcus }\end{array}$ \\
\hline A.dorsata & TNAU & Pollen 1 & $\begin{array}{c}14.10 \pm 7.51 \\
(n=3)\end{array}$ & Flat & $\begin{array}{l}\text { Shinny } \\
\text { surface }\end{array}$ & Smooth & whitish & Cocci in tetrads & Micrococcus \\
\hline T. iridipennis & TNAU & Pollen 1 & $\begin{array}{c}5.14 \pm 1.56 \\
(n=3)\end{array}$ & Raised & $\begin{array}{l}\text { Shinny } \\
\text { mucoid } \\
\text { surface }\end{array}$ & Smooth & Creamy & Cocci in chains & $\begin{array}{c}\text { Enterococcus } \\
\text { Streptococcus } \\
\text { Pediococcus }\end{array}$ \\
\hline
\end{tabular}

(Mean \pm SE of 3 replicates; SE, standard error; CFU, colony forming units) 
Table.4 Population and colony morphology of LAB in the bee bread of honey bees

\begin{tabular}{|c|c|c|c|c|c|c|c|c|c|}
\hline \multirow[t]{2}{*}{ Bee species } & \multirow{2}{*}{$\begin{array}{l}\text { Place of } \\
\text { collection }\end{array}$} & \multirow[t]{2}{*}{ Number of isolates } & \multirow{2}{*}{$\begin{array}{c}\text { LAB } \\
\left(10^{5} \mathrm{CFU} / \mathrm{ml}\right)\end{array}$} & \multicolumn{4}{|c|}{ Colony morphology } & \multirow{2}{*}{$\begin{array}{l}\text { Cell } \\
\text { shape }\end{array}$} & \multirow{2}{*}{$\begin{array}{l}\text { Probable LAB } \\
\text { genera }\end{array}$} \\
\hline & & & & Elevation & $\begin{array}{l}\text { Colony } \\
\text { texture }\end{array}$ & Edge & $\begin{array}{l}\text { Colony } \\
\text { colour }\end{array}$ & & \\
\hline \multirow[t]{3}{*}{ A. ceranaindica } & \multirow[t]{3}{*}{ TNAU } & Bee bread 1 & $\begin{array}{c}25.15 \pm 5.10 \\
(\mathrm{n}=3)\end{array}$ & Flat & $\begin{array}{l}\text { Coarse } \\
\text { surface }\end{array}$ & Rough & Creamy & Cocci in singles & $\begin{array}{l}\text { Enterococcus } \\
\text { Lactococcus } \\
\text { Leuconostoc }\end{array}$ \\
\hline & & Bee bread 2 & $\begin{array}{l}19.75 \pm 7.23 \\
\quad(\mathrm{n}=3)\end{array}$ & Raised & $\begin{array}{l}\text { Shinny } \\
\text { mucoid } \\
\text { surface }\end{array}$ & Smooth & Creamy & Cocci in chains & $\begin{array}{l}\text { Enterococcus } \\
\text { Streptococcus } \\
\text { Pediococcus }\end{array}$ \\
\hline & & Bee bread 3 & $\begin{array}{c}10.32 \pm 4.67 \\
(\mathrm{n}=3)\end{array}$ & Flat & $\begin{array}{l}\text { Shinny } \\
\text { surface }\end{array}$ & Smooth & whitish & Cocci in tetrads & Micrococcus \\
\hline \multirow[t]{2}{*}{ A. mellifera } & \multirow[t]{2}{*}{ TNAU } & Bee bread 1 & $\begin{array}{c}17.83 \pm 5.43 \\
(\mathrm{n}=3)\end{array}$ & Flat & $\begin{array}{l}\text { Coarse } \\
\text { surface }\end{array}$ & Rough & Creamy & Cocci in singles & $\begin{array}{c}\text { Enterococcus } \\
\text { Lactococcus } \\
\text { Leuconostoc }\end{array}$ \\
\hline & & Bee bread 2 & $\begin{array}{c}10.56 \pm 3.90 \\
(\mathrm{n}=3)\end{array}$ & Raised & $\begin{array}{l}\text { Shinny } \\
\text { mucoid } \\
\text { surface }\end{array}$ & Smooth & $\begin{array}{l}\text { Yellowish } \\
\text { surround }\end{array}$ & Cocci in chains & $\begin{array}{l}\text { Enterococcus } \\
\text { Streptococcus } \\
\text { Pediococcus }\end{array}$ \\
\hline \multirow[t]{2}{*}{ A. florea } & \multirow[t]{2}{*}{ TNAU } & Bee bread 1 & $\begin{array}{c}15.31 \pm 5.71 \\
(\mathrm{n}=3)\end{array}$ & Flat & $\begin{array}{l}\text { Coarse } \\
\text { surface }\end{array}$ & Rough & Creamy & Cocci in singles & $\begin{array}{l}\text { Enterococcus } \\
\text { Lactococcus } \\
\text { Leuconostoc }\end{array}$ \\
\hline & & Bee bread 2 & $\begin{array}{c}9.11 \pm 1.37 \\
(\mathrm{n}=3)\end{array}$ & Raised & $\begin{array}{l}\text { Shinny } \\
\text { mucoid } \\
\text { surface }\end{array}$ & Smooth & Creamy & Cocci in chains & $\begin{array}{c}\text { Enterococcus } \\
\text { Streptococcus } \\
\text { Pediococcus }\end{array}$ \\
\hline A. dorsata & TNAU & Bee bread 1 & $\begin{array}{c}21.32 \pm 6.63 \\
(\mathrm{n}=3)\end{array}$ & Flat & $\begin{array}{l}\text { Shinny } \\
\text { surface }\end{array}$ & Smooth & whitish & Cocci in tetrads & Micrococcus \\
\hline T. iridipennis & TNAU & Bee bread 1 & $\begin{array}{c}13.05 \pm 2.80 \\
(n=3)\end{array}$ & Flat & $\begin{array}{l}\text { Shinny } \\
\text { surface }\end{array}$ & Smooth & whitish & Cocci in tetrads & Micrococcus \\
\hline
\end{tabular}

(Mean \pm SE of 3 replicates; SE, standard error; $\mathrm{CFU}$, colony forming units)

Table.5 Population and colony morphology of LAB in the royal jelly

\begin{tabular}{|c|c|c|c|c|c|c|c|c|c|c|}
\hline \multirow[t]{2}{*}{ Bee species } & \multirow[t]{2}{*}{ Place of collection } & \multirow[t]{2}{*}{ Isolation source } & \multirow[t]{2}{*}{ Number of isolates } & \multirow{2}{*}{$\begin{array}{c}\text { LAB } \\
\left(10^{5} \mathrm{CFU} / \mathrm{ml}\right)\end{array}$} & \multicolumn{4}{|c|}{ Colony morphology } & \multirow[t]{2}{*}{ Cell shape } & \multirow{2}{*}{$\begin{array}{c}\text { Probable } \\
\text { LAB genera }\end{array}$} \\
\hline & & & & & Elevation & $\begin{array}{l}\text { Colony } \\
\text { texture }\end{array}$ & Edge & $\begin{array}{l}\text { Colony } \\
\text { colour }\end{array}$ & & \\
\hline A. ceranaindica & TNAU & Queen cell cup & Royal jelly 1 & $\begin{array}{c}10.23 \pm 2.19 \\
(\mathrm{n}=3)\end{array}$ & Raised & $\begin{array}{l}\text { Shinny } \\
\text { mucoid } \\
\text { surface }\end{array}$ & Smooth & Creamy & $\begin{array}{l}\text { Cocci in } \\
\text { chains }\end{array}$ & $\begin{array}{c}\text { Enterococcus } \\
\text { Streptococcus } \\
\text { Pediococcus }\end{array}$ \\
\hline A. ceranaindica & TNAU & $\begin{array}{l}\text { Worker bee } \\
\text { head }\end{array}$ & Royal jelly 1 & $\begin{array}{c}14.30 \pm 3.13 \\
(\mathrm{n}=3)\end{array}$ & Flat & $\begin{array}{l}\text { Shinny } \\
\text { surface }\end{array}$ & Smooth & whitish & $\begin{array}{l}\text { Cocci in } \\
\text { tetrads }\end{array}$ & Micrococcus \\
\hline A. mellifera & Dhalavaipuram & $\begin{array}{l}\text { Commercial bee } \\
\text { product }\end{array}$ & Royal jelly 1 & $\begin{array}{c}20.15 \pm 2.50 \\
(\mathrm{n}=3)\end{array}$ & Flat & $\begin{array}{l}\text { Shinny } \\
\text { surface }\end{array}$ & Smooth & whitish & $\begin{array}{l}\text { Cocci in } \\
\text { tetrads }\end{array}$ & Micrococcus \\
\hline
\end{tabular}

(Mean \pm SE; SE, standard error; $n=$ number of replicates; CFU, colony forming units) 


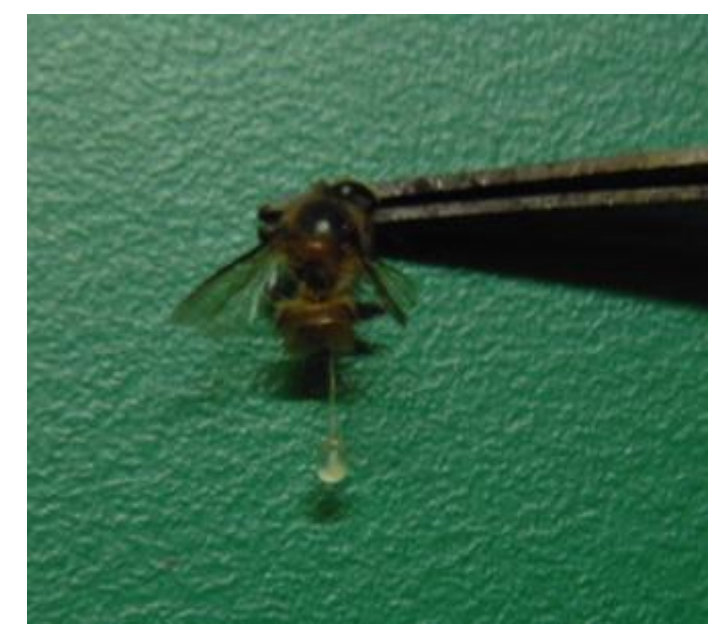

Plate.1 Detached honey stomach (crop) of $A$. ceranaindica worker bee.

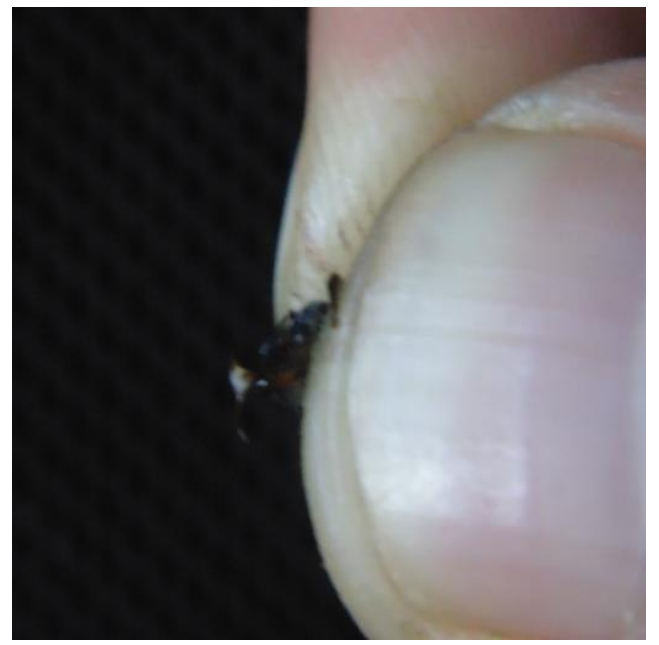

Plate.3 Extracting royal jelly by pressing the head of an A. ceranaindica nurse bee.

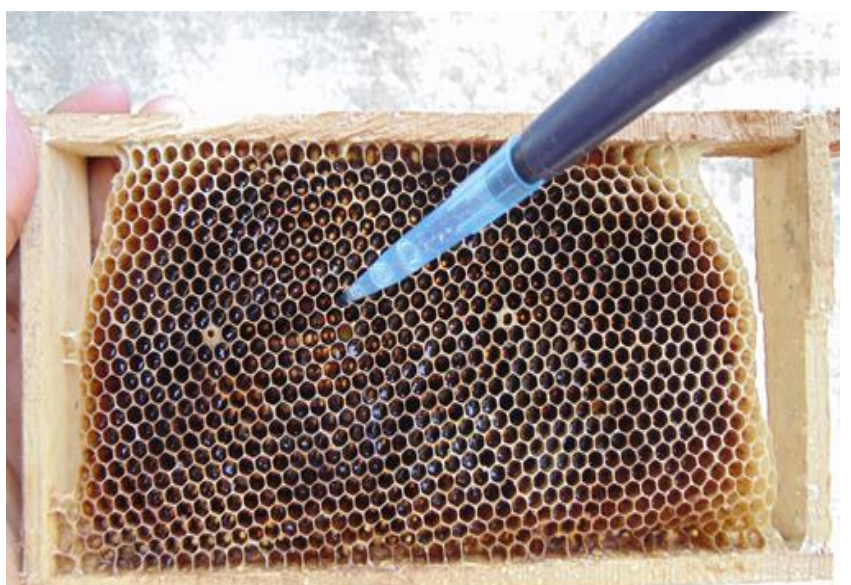

Plate.2 Collection of unprocessed honey in A. ceranaindica.

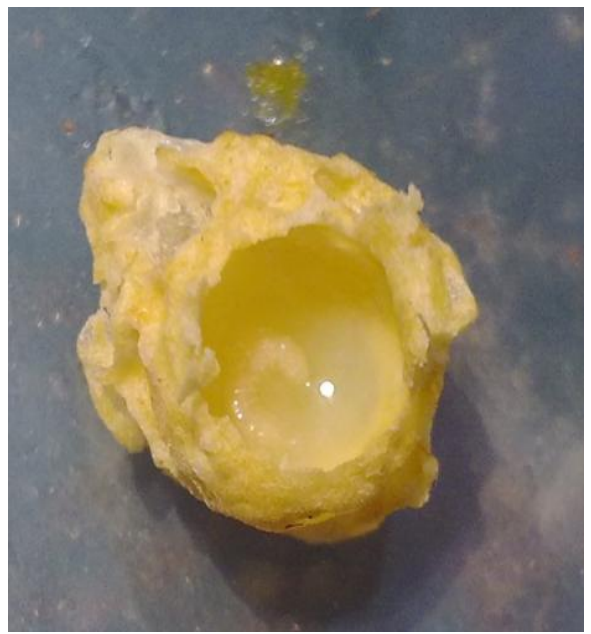

Plate.4 Royal jelly of $A$. ceranaindica in a queen cell cup before scooping it out.

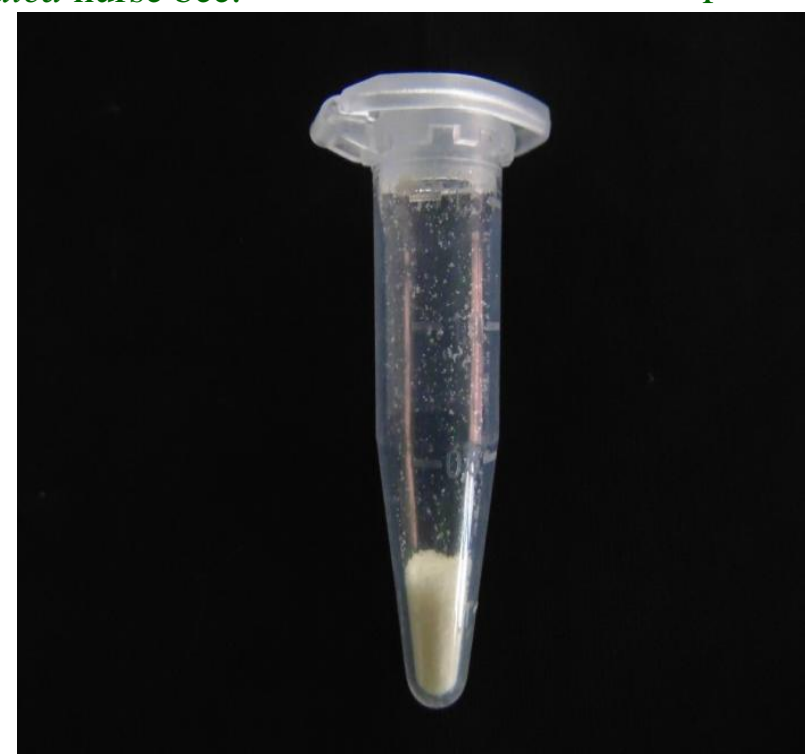

Plate.5 Commercial sample of $A$. melliefra royal jelly (bee product) 


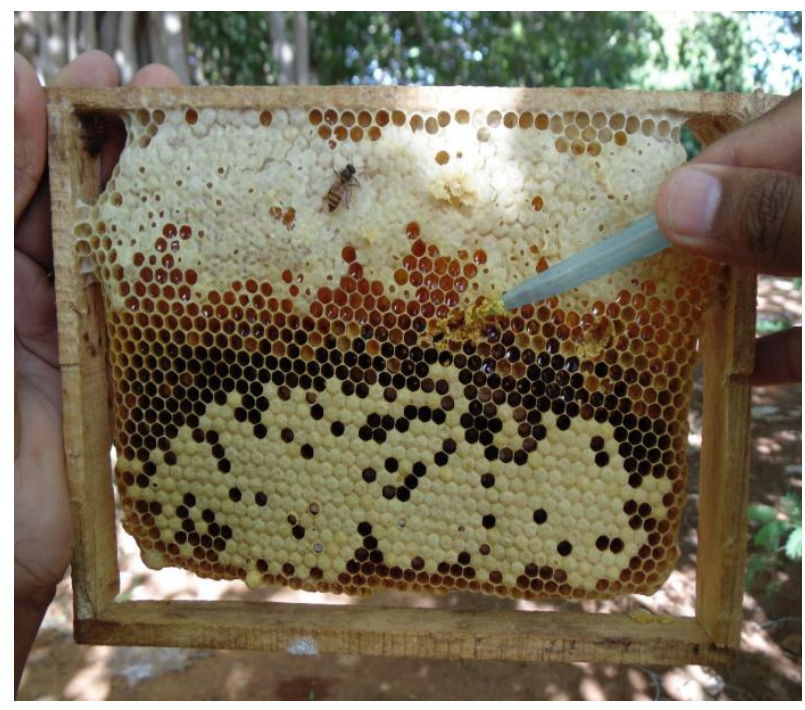

Plate.6 Collecting the bee bread from an $A$. ceranaindica comb.

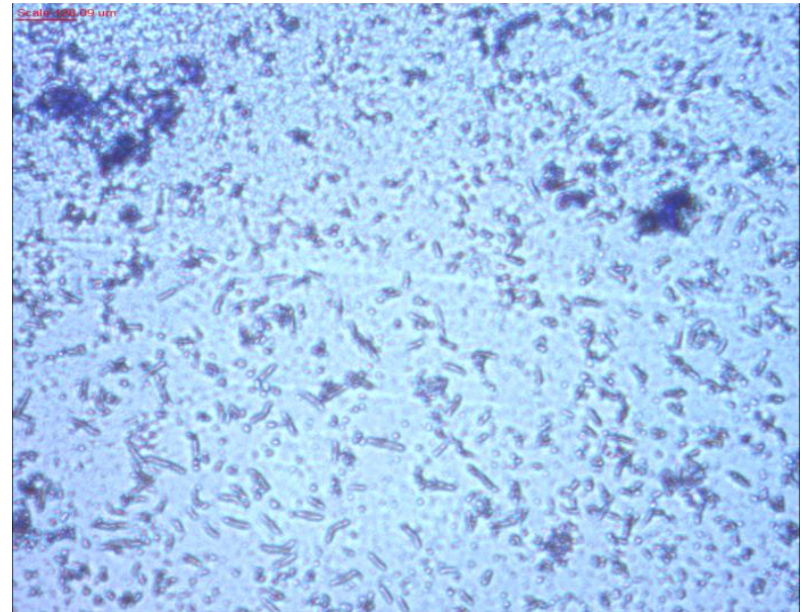

Plate.8 LAB in the honey stomach of $A$. ceranaindica worker bees. Cells were gram positive cocci and rods.

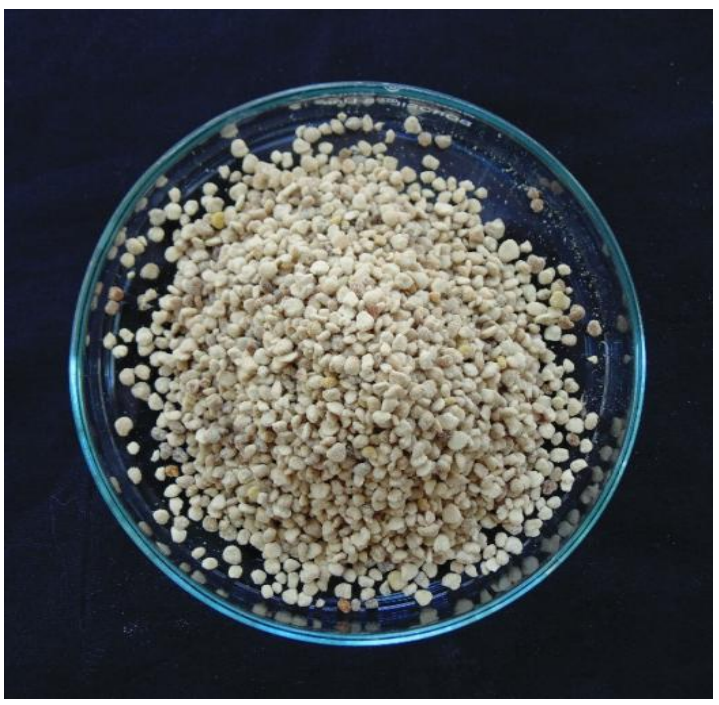

Plate.7 Commercial bee pollen of A. mellifera.

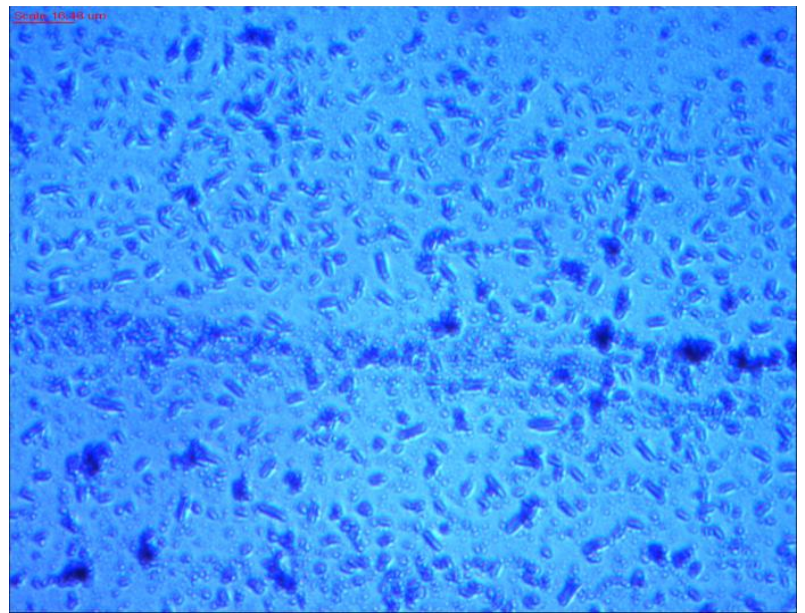

Plate.9 LAB in the honey bee stomach of $A$. ceranaindica worker bees. Cells were gram Positive cocci in singles and chains.

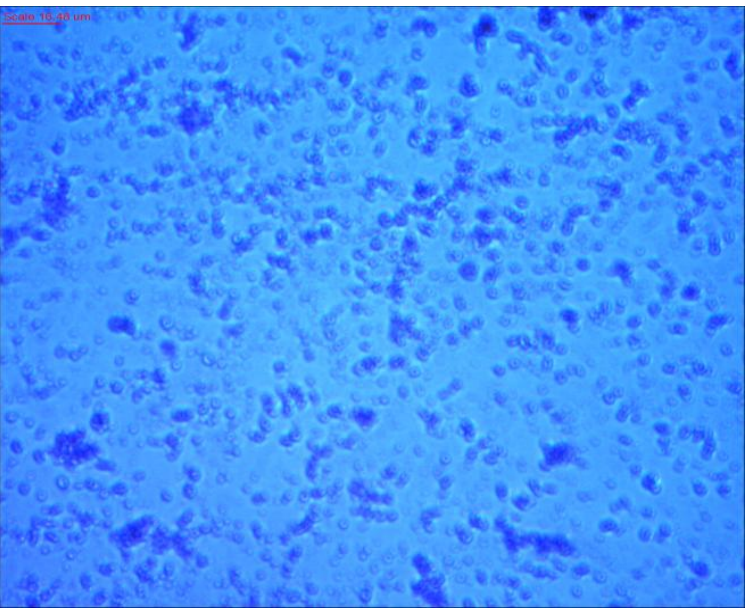

Plate.10 LAB in the honey stomach of $A$. ceranaindica worker bees. Cells were gram-positive cocci in tetrads 
They also end up as residues in honey (Johnson and Jadon, 2010). The antibiotics may also kill the beneficial honey bee microflora that is part of the natural honey bee defence against pathogens, including probiotics (Yoshiama and Kimura, 2009), which produce antimicrobials (Hamdi et al., 2011). Thus stress management strategies in honey bees need to focus on non-chemical and non-antibiotic measures such as probiotics (Moritz et al., 2010). The results of this survey highlights the diversity of LAB associated with honey bees and their environment. As a non-chemical measure, dusting the bees with powdered table sugar has been reported to help them dislodge the mites by enhanced grooming (Fakhimzadeh, 2001; Conrad, 2013). Recently, it has been reported that powdered sugar acts a reservoir of probiotic LAB, probably suppressing diseases in bees through augmentation of LAB populations (Mathialagan, 2014; Mathialagan et al., 2015). Prophylactic feeding of LAB isolated from the bee world will also help the bees resist the diseases from enhanced immunity.

Varroa infestation and both viral and bacterial diseases are key stressors in beekeeping that need urgent attention by the beekeepers in South India. In this background this investigation explored the diversity of LAB genera such as Enterococcus, Lactobacillus, Micrococcus, Lactococcus, Streptococcus, Pediococcus, and Leconostoc from the honey stomach, honey, bee pollen, bee bread and royal jelly collected from different bee species. However, these isolates need to be characterized and confirmed through molecular studies in future. Antibiotics, besides residue in honey, may cause elimination of symbiotic gut microbiota, or microbial dysbiosis. Key among them is the probiotic LAB that are significantly abundant in and around bees, overwhelmingly related to the well-being of honey bees. The symbiotic
$\mathrm{AAB}$ are also likely to occur in association with LAB in honey bees. While the ecto parasitic Varroa mites and the diseases they transmit need to be controlled without pesticides and antibiotics, enriching the gut microbiota with prophylactic feeding of potential LAB strains as a bee feed will help the bees manage the stress from diseases.

\section{Acknowledgment}

We are grateful to the Department of Agricultural Entomology and Microbiology, Tamil Nadu Agricultural University, Coimbatore for providing laboratory facilities. This work was financially supported by the University Grants Commission, Rajiv Gandhi National Fellowship, New Delhi.

\section{References}

Awan, J. A., Rahman, S. U., 2005. Microbiology Manual. Unitech Communications, Faisalabad, Pakistan, pp 49-51.

Aween, M. M., Hassan, Z., Muhialdin, B.J., Noor, H. M., Eljamel, Y. A., 2012. Evaluation on antibacterial activity of Lactobacillus acidophilus strains isolated from honey. American Journal of Applied Science 9 (6), 807-817.

Axelsson, L., 1998. Lactic Acid Bacteria: Classification and Physiology. In: Lactic Acid Bacteria, Microbiology and Functional Aspects. Ed. S. Salminen and A. von Wright, Marcel Decker Inc, New York, USA.

Berry, J. A., Hood, W.M., Pietravalle, S., Delaplane, K.S., 2013.Field-level sublethal effects of approved bee hive chemicals on honey bees (Apis mellifera L). PLOS ONE 8 (10), e76536.

Conrad, R., 2013. Natural Beekeeping: Organic Approaches to Modern Apiculture. Chelsea Green Publisher. 304 pp.

Corby-Harris V., Maes, P., Anderson, K. E., 2014. The bacterial communities associated with honey bee (Apis mellifera) foragers. PLOS ONE9 (4), e95056. 
Crotti, E., Rizzi, A., Chouaia, B., Ricci, I., Favia, G., Alma, A., Sacchi, L., Bourtzis, K., Mandrioli, M., Cherif, A., Bandi, C., D. Daffonchio, D.,2010. Acetic Acid Bacteria, newly emerging symbionts of insects. Applied Environmental Microbiology 76 (21), 6963-6970.

Ellis, J.D., Evans, J.D., Pettis, J., 2010. Colony losses, managed colony population decline, and Colony Collapse Disorder in the United States, Journal of Apicultural Research 49 (1), 134-136.

Endo, A., Irisawa, T., Futagawa-Endo, Y., Takano, K., du Toit, M., Okada, S., Dicks, L.M.T., 2012.Charactreization and emended description of Lactobacillus kunkeei as a frutophilic lactic acid bacterium. International Journal of Systematic and Evolutionary Microbiology 62 (3), 500-504.

Fakhimzadeh, K., 2001. Powdered sugar dusting for the control of varroosis. In: Proc. 37th Int. Apic. Cong., 28 October - 1 November 2001, Durban, South Africa.

FAO/WHO., 2001. Health and nutritional properties of probiotics in food including powder milk with lactic acid bacteria, a joint FAO/WHO expert consultation Cordoba, Argentina.

Forsgren, E., Olofsson, T.C., A. Vasquez, A., Fries, I., 2009. Novel lactic acid bacteria inhibiting Paenibacillus larvae in honey bee larvae. Apidologie 41, 99-108.

Hamdi, C., Balloi, A., Essanaa, J., Crotti, E., Gonella, E., Raddadi, N., Ricci, I., Boudabous, A., Borin, S., Manino, A., Bandi, C., Alma, A., Daffonchio, D., Cherif, A., 2011. Gut microbiomedysbiosis and honey bee health. Journal of Applied Entomology135, 524-533.

Hammes, W.P., Weiss, N., Holzaptel, W., 1992. The genera Lactobacillus and Carnobacterium; In Albert, B., Truper, H.G., Dworkin, M., Harder, W., Schleifer, H.K., ed.: Prokayotes. 2nd Eds., Vol. II.New York Inc. Springer-Verlag.

Harrigan, W. F., McCance, M. E., 1976. Laboratory Methods in Food and Dairy Microbiology. Academic Press, London, UK.
Holzapfel, W. H., Wood, B.J.B., 2014. Lactic Acid Bacteria: Biodiversity and Taxonomy. John Wiley \& Sons Ltd. 632 pp.

Hosny, I.M., El-Ghani, S. A., Nadir, A. S., 2009. Nutrient composition and microbiological quality of three unifloral honeys with emphasis on processing of honey probiotic yoghurt. Global Veterinaria3, 107-112.

Johnson, S., Jadon N., 2010.Antibiotic residues in honey. Centre for Science and Environment, New Delhi. pp. 48.

Kandler, O., Weiss, N., 1986. Regular, nonsporing Gram-positive rods. In Sneath, P.H., Mair, N., Sharpe, M.E. Holt, J.G., ed. Bergey's Manual of systematic bacteriology. Baltimore. Williams and Wilkins 11, 1208-1234.

Lebreton, F., Willems, R.J.L., Gilmore, M.S., 2014. Enterococci: From Commensals to Leading Causes of Drug Resistant Infection (Internet). Enterococcus Diversity, Origins in Nature, and Gut Colonization. Boston: Massachusetts Eye and Ear Infirmary 1-41.

Liu, W., Pang, H., Zhang, H., Cai, Y., 2014. Lactic Acid Bacteria Fundamentals and Practice. Biodiversity of Lactic Acid Bacteria (LAB). 535 pp. DOI: 10.1007/97894-017-8841-0-2.

Mathialagan, M., 2014. Probiotic lactic acid bacteria (LAB) for Varroa-associated stress management in honey bees. M. Sc. (Ag.) Thesis (Unpublished), Tamil Nadu Agricultural University, Coimbatore, India.232 pp.

Mathialagan, M., Johnson Thangaraj Edward, Y.S., David, P.M.M., Srinivasan, M.R., Mohankumar, S., Senthilkumar, M., 2017. A survey in Tamil Nadu of Varroajacobsoni (Oudemans) ectoparasitic on Indian honey bees, Apiscerana F. Journal of Entomology and Zoology Studies 5 (6), 190-200.

Mathialagan, M., Sabarinathan, K.G., Muthuraman, M., David, P.M.M., 2015. Effect of dusting powdered sugar and of feeding probiotic lactic acid bacteria (LAB) on varroosis in Apiscerana indica. International Conference on Innovative Insect Management Approaches for Sustainable Agro Eco System. Madurai, January 27-30, 672-673. 
Moritz, R.F.A., Miranda, J., Fries, I., Conte, Y.L., Neumann, P., Paxton, R.J., 2010. Research strategies to improve honey bee health in Europe. Apidologie41, 227-242.

Pattabhiramaiah, M., Reddy, M. S., Brueckner, D., 2012. Detection of novel probiotic bacterium Lactobacillus spp. in the workers of Indian honey bee, Apiscerana indica. International Journal of Environmental Science 2 (3), 1135-1143.

Rangberg, A., Diep, D.B., Rudi, K., Amdam, G.V., 2012. Paratransgenesis: an approach to improve colony health and molecular insight in honey bees (Apis mellifera). Integrative Comparative Biology 52 (1), 89-99.

Raoa, K.M., Katna, S., Rana, B.S., Rana, R., 2016. Thai sacbrood and sacbrood viruses versus European foulbrood of hive bees in India - a review. Journal of Apicultural Research 1-8. DOI: 10.1080/00218839.2016.1145417.

Savadogo, A., Quattara, C.A., Bassole, H.N., Traore, S.A., 2006. Bacteriocins and Lactic acid bacteria a mini review. African Journal of Biotechnology 5 (9), 678-684.

Sharma, K., 2007. Manual of microbiology: tools and techniques, $2^{\text {nd }}$ ed. Ane books Pvt. Ltd, New Delhi, 282 pp.

Stiles, M. E., Holzapfel, W.H., 1997.Lactic acid bacteria of foods and their current taxonomy. International Journal of Food Microbiology36 (1), 1-29.
Tajabadi, N., Mardan, M., Manap, M.Y.A. Shuhaimi, M., Meimandipour, A., Nategghi, L., 2011. Detection and identification of Lactobacillus bacteria found in the honey stomach of the giant honey bee Apisdorsata. Apidologie42 (5), 642-649.

Thompson, H.M., Brown, M.A., Ball, R.F., Bew, M.H., 2002. First report Varroa destructor resistance to pyrethroids in the UK. Apidiologie. 33, 357-366.

Vasquez, A., Forsgren, E., Fries, I., Paxton, R. J., Flaberg, E., 2012. Symbionts as major modulators of insect health: lactic acid bacteria and honey bees. PLOS ONE 7 (3), e33188.

Vojvodic, S., Rehan, S.M., Anderson, K.E., 2013.Microbial gut diversity of Africanized and European honey bee larval instars. PLOS ONE 8 (8), e72106.

Wongchai, V., Ratanavalachai, T., 2002. Seasonal variation of chemical composition of royal jelly produced in Thailand. International Journal of Science and Technology 7 (2), 18.

Yoshiama, M., Kimura, K., 2009. Bacteria in the gut of Japanese honey bee, Apiscerana japonica, and their antagonistic effect against Paenibacillus larvae, the causal agent of American Foul Brood. Journal of Invertebrate Pathology 102, 91-96.

\section{How to cite this article:}

Mathialagan, M., Y.S. Johnson Thangaraj Edward, P.M.M. David, M. Senthilkumar, M.R. Srinivasan and Mohankumar, S. 2018. Isolation, Characterization and Identification of Probiotic Lactic Acid Bacteria (LAB) from Honey Bees. Int.J.Curr.Microbiol.App.Sci. 7(04): 894-906. doi: https://doi.org/10.20546/ijcmas.2018.704.096 